PROCEEDINGS OF THE

AMERICAN MATHEMATICAL SOCIETY

Volume 128, Number 12, Pages 3475-3482

S 0002-9939(00)05906-2

Article electronically published on July 27, 2000

\title{
RANDOM ITERATIONS OF HOLOMORPHIC MAPS IN COMPLEX BANACH SPACES
}

\author{
K. WŁODARCZYK, D. KLIM, AND E. GONTAREK
}

(Communicated by Dale Alspach)

\begin{abstract}
Conditions guaranteeing the uniform convergence to constant maps of random iterations of holomorphic contractions on unbounded domains in complex Banach spaces are established.
\end{abstract}

\section{INTRODUCTION}

The fundamental problem which arises in several areas of mathematics and applications is to understand the behaviour of high random iterations $f_{1} \circ f_{2} \circ \cdots \circ f_{n}$ or $f_{n} \circ f_{n-1} \circ \cdots \circ f_{1}$ and iterations $f^{[n]}=f \circ f \circ \cdots \circ f(n$ times $), n \in \mathbb{N}$, where $f, f_{n}$, $n \in \mathbb{N}$, are self-maps of domains in some spaces (e.g. see [1]-26] and references therein). Special cases of this type of iterations appear, for example, in fixed point theory, the theory of differential equations, functional equations, numerical analysis, perturbation theory, fractal geometry, computer graphics, population biology, etc.

It is a well-known problem to determine conditions guaranteeing the uniform convergence to constant maps of random iterations $f_{1} \circ f_{2} \circ \cdots \circ f_{n}, n \in \mathbb{N}$, where $f_{n}$, $n \in \mathbb{N}$, are holomorphic self-maps of domains in complex spaces. The solution of this problem is known for an important but special class of domains in finite-dimensional complex spaces $\mathbb{C}$ and $\mathbb{C}^{n}$ and appeared in [1, 2, 8, 9, 10, 11, 15, 26] (see also references therein). The proofs in the papers cited above are crucially based on: the Riemann mapping theorem, the adaptation of Henrici's technique, the Wolff-Denjoy theorem, the properties of the modulus of equicontinuity of maps or the inequality $K_{G}(z, w) \geq K_{B(z, R)}(z, w)=\omega(0,\|w-z\| / R) \geq\|w-z\| / R, z, w \in G$, when $G$ is bounded, where $K_{G}$ is the Kobayashi distance of $G, \omega$ denotes the Poincaré distance on the unit disc in $\mathbb{C}$ and $G \subset B(z, R)=\left\{w \in \mathbb{C}^{n}:\|w-z\|<R\right\}$. However, the situation is quite different if random iterations are studied on unbounded domains contained in infinite-dimensional complex spaces and, in such a case, it remains an open question whether random iterations of holomorphic self-maps converge uniformly to constant maps. In the present paper we give conditions guaranteeing the uniform convergence to constant maps of random iterations of holomorphic selfmaps of domains in complex Banach spaces under a framework where the space is not assumed to be finite-dimensional or the domain to be bounded. Theorem 2.1

Received by the editors May 4, 1998.

2000 Mathematics Subject Classification. Primary 46G20, 32H50.

Key words and phrases. Random iterations, holomorphic maps, unbounded domains, complex Banach spaces. 
of this paper includes the fixed point theorem of C. J. Earle and R. S. Hamilton [6 and the result from W. J. Zhang and F. J. Ren [26, Theorem 2.1]. The methods of proving Theorem 2.1 differ from those given by the above-mentioned authors. For details, see Section 4

\section{Statement of Result}

The statement of the theorem requires a definition. Let $E$ be a complex Banach space. Let $G$ be a nonempty domain (an open and connected set) contained in $E$. We say that a set $D \subset E$ lies strictly inside $G$ if $\operatorname{dist}[D, E \backslash G]>0$.

We show

Theorem 2.1. Let $G$ be a nonempty domain contained in a complex Banach space $E$. Let a subset $D$ of $G$ be bounded, and suppose that $D$ lies strictly inside $G$. Let $\mathcal{H}_{G, D}=\{f \in \operatorname{Hol}(G, E): f(G) \subset D\}$, and suppose that one of the following cases holds:

(a) $G$ is convex.

(b) $D$ is star-shaped (in particular, convex).

(c) $D$ is connected and contained in a compact subset of $G$.

Then, for each sequence $\left\{f_{n}\right\}$ from $\mathcal{H}_{G, D}$, there exists a point $w \in G$ such that the sequence $\left\{F_{n}\right\}, F_{n}=f_{1} \circ f_{2} \circ \cdots \circ f_{n}, n \in \mathbb{N}$, converges to the constant map $x \mapsto w$ uniformly on $G$.

Maps belonging to the family $\mathcal{H}_{G, D}$ are called holomorphic contractions.

\section{Proof of Theorem 2.1}

Let $\left\{f_{n}\right\}$ be an arbitrary and fixed sequence from $\mathcal{H}_{G, D}$ and let $F_{n}=f_{1} \circ f_{2} \circ$ $\cdots \circ f_{n}, n \in \mathbb{N}$.

Denote by $H^{\infty}(G)$ the space of holomorphic maps $g: G \rightarrow \mathbb{C}$ such that $\sup \{|g(x)|$ : $x \in G\}<+\infty$. Then $H^{\infty}(G)$ is a normed vector space with respect to the norm $\|g\|=\sup \{|g(x)|: x \in G\}$. Denote by $H^{\infty}(G)^{*}$ its topological dual space.

The evaluation map $\varphi: G \rightarrow H^{\infty}(G)^{*}$ is defined by

$$
\varphi(x)(g)=g(x) \text { for } x \in G \text { and } g \in H^{\infty}(G) .
$$

We prove that the map $\varphi: G \rightarrow H^{\infty}(G)^{*}$ is holomorphic in $G$ and

$$
\varphi^{\prime}(x)(v)(g)=g^{\prime}(x)(v) \quad \text { for }(x, v) \in G \times E, g \in H^{\infty}(G) .
$$

Indeed, let $x \in G$ be arbitrary and fixed and let $B$ be a balanced neighborhood of zero of the form $B=\{v \in E: x+\lambda v \in G,|\lambda| \leq 1\}$. If $v \in B$, then $x+\{\lambda v:|\lambda| \leq 1\}$ is a compact subset of $G$ and hence there exists $R>1$ such that $x+\{\lambda v:|\lambda| \leq$ $R\} \subset G$. By holomorphy, $\varphi(x+v)(g)=g(x+v)=\sum_{m=0}^{\infty} P_{m, x}(v)(g), g \in H^{\infty}(G)$, where, for every positive integer $m$, by the Cauchy integral formula,

$$
P_{m, x}(v)(g)=\frac{1}{2 \pi i} \int_{|\lambda|=R} \frac{g(x+\lambda v)}{\lambda^{m+1}} d \lambda, \quad g \in H^{\infty}(G) .
$$

From the above we obtain $\left|P_{m, x}(v)(g)\right| \leq\|g\| R^{-m}, g \in H^{\infty}(G)$, where, by definition, $\|g\|=\sup \{|g(y)|: y \in G\}<+\infty$ and, consequently, $\left\|P_{m, x}(v)\right\| \leq R^{-m}$, 
$m \in \mathbb{N}$. Now, let us observe that

$$
\begin{aligned}
\left\|\varphi(x+v)-\sum_{m=0}^{n} P_{m, x}(v)\right\| \\
=\sup \left\{\left|\left[\varphi(x+v)-\sum_{m=0}^{n} P_{m, x}(v)\right](g)\right|:\|g\| \leq 1, g \in H^{\infty}(G)\right\} \\
=\sup \left\{\left|\sum_{m=n+1}^{\infty} P_{m, x}(v)(g)\right|:\|g\| \leq 1, g \in H^{\infty}(G)\right\} \\
\leq \sum_{m=n+1}^{\infty} \sup \left\{\left|P_{m, x}(v)(g)\right|:\|g\| \leq 1, g \in H^{\infty}(G)\right\} \\
=\sum_{m=n+1}^{\infty}\left\|P_{m, x}(v)\right\| \leq \sum_{m=n+1}^{\infty} R^{-m} .
\end{aligned}
$$

Hence $\varphi(x+v)=\sum_{m=0}^{\infty} P_{m, x}(v)$ for each $v \in B$. Thus $\varphi$ is holomorphic and, moreover, the formula $\varphi^{\prime}(x)(v)(g)=P_{1, x}(v)(g)=g^{\prime}(x)(v)$ for $(x, v) \in G \times E$, $g \in H^{\infty}(G)$, holds and (3.1) is thus proved.

Let $\alpha_{G}: G \times E \rightarrow \mathbb{R}_{+}$be a map of the form

$$
\alpha_{G}(x, v)=\left\|\varphi^{\prime}(x)(v)\right\|, \quad(x, v) \in G \times E,
$$

and let $\rho_{G}$ be the integrated form of $\alpha_{G}$, i.e.

$$
\rho_{G}(a, b)=\inf _{\gamma} L(\gamma), \quad L(\gamma)=\int_{0}^{1} \alpha_{G}\left[\gamma(t), \gamma^{\prime}(t)\right] d t
$$

for all $a, b \in G$, where the infimum is taken over all curves $\gamma:\langle 0,1\rangle \rightarrow G$ with piecewise continuous derivatives, such that $\gamma(0)=a, \gamma(1)=b$. The map $\rho_{G}$ is pseudometric on $G$. We call $\rho_{G}$ the Carathéodory-Reiffen-Finsler pseudometric on $G$. For details, see [6] and [14].

We prove that if one of assumptions (a)-(c) holds, then

$$
M_{D}=\sup \left\{\rho_{G}(x, y): x, y \in D\right\}<+\infty .
$$

Indeed, in case (a), the set $G$ is convex and $D$ lies strictly inside $G$, and then $\operatorname{co}(D)$ (co stands for the convex hull operator) also lies strictly inside $G$, and $M_{D} \leq$ $M_{\mathrm{co}(D)}$. Let $\varepsilon>0$ be such that $u+B(\varepsilon) \subset G$ for all $u \in M_{\operatorname{co}(D)}$, where $B(\varepsilon)=\{x \in$ $E:\|x\| \leq \varepsilon\}$. Let $g \in H^{\infty}(G)$ with $\|g\| \leq 1$. If $h \in E \backslash\{0\}$, then $\varepsilon h /\|h\| \in B(\varepsilon)$ and, for $u \in \operatorname{co}(D)$, by the Cauchy integral formula,

$$
\left|g^{\prime}(u)(h /\|h\|)\right|=\left|(2 \pi i)^{-1} \int_{|\lambda|=\varepsilon} g(u+\lambda h /\|h\|) \lambda^{-2} d \lambda\right| \leq \sup _{v \in G}|g(v)| \leq 1 .
$$

Therefore

$$
\left|g^{\prime}(u)(h)\right| \leq\|h\| \quad \text { for all } h \in E \text { and } u \in \operatorname{co}(D) .
$$

For any $x, y \in \operatorname{co}(D)$, there exists $\gamma:\langle 0,1\rangle \rightarrow \operatorname{co}(D)$ defined by the formula $\gamma(t)=t x+(1-t) y$. By (3.3), we obtain

$$
\begin{aligned}
L(\gamma) & =\int_{0}^{1} \alpha_{G}\left[\gamma(t), \gamma^{\prime}(t)\right] d t=\int_{0}^{1}\left\|\varphi^{\prime}[t x+(1-t) y](y-x)\right\| d t \\
& \leq \sup \left\{\left\|\varphi^{\prime}[t x+(1-t) y](y-x)\right\|: t \in\langle 0,1\rangle\right\} .
\end{aligned}
$$


However,

$$
\begin{aligned}
\left\|\varphi^{\prime}(u)(v)\right\| & =\sup \left\{\left|\varphi^{\prime}(u)(v)(g)\right|: g \in H^{\infty}(G),\|g\| \leq 1\right\} \\
& =\sup \left\{\left|g^{\prime}(u)(v)\right|: g \in H^{\infty}(G),\|g\| \leq 1\right\}, \quad(u, v) \in G \times E .
\end{aligned}
$$

From (3.5)- (3.7) we obtain $L(\gamma) \leq \sup \left\{\left|g^{\prime}[t x+(1-t) y](y-x)\right|: t \in\langle 0,1\rangle, g \in\right.$ $\left.H^{\infty}(G),\|g\| \leq 1\right\} \leq\|y-x\|$. By our assumptions and the definition of $\rho_{G}$, if $x, y \in \operatorname{co}(D)$, then $\rho_{G}(x, y) \leq L(\gamma) \leq\|y-x\| \leq \operatorname{diam}(\operatorname{co}(D))$ and thus $M_{D}<+\infty$.

(b) Let $D$ be star-shaped with respect to $s \in D$. For any $x_{i} \in D$, there exist $\gamma_{i}:\langle 0,1\rangle \rightarrow D$ defined by the formulae $\gamma_{i}(t)=t s+(1-t) x_{i}, i=1,2$. By the assumptions and the definition of $\rho_{G}$, if $x_{1}, x_{2} \in D$, then $\rho_{G}\left(x_{1}, x_{2}\right) \leq \sum_{i=1}^{2} L\left(\gamma_{i}\right) \leq$ $\sum_{i=1}^{2}\left\|s-x_{i}\right\|$ and thus $M_{D} \leq \sup \left\{L\left(\gamma_{1}\right)+L\left(\gamma_{2}\right): x_{1}, x_{2} \in D\right\} \leq 2 \operatorname{diam}(D)<+\infty$.

(c) In this case, there exist $a, b \in \bar{D}$ such that $M_{D}=\sup \left\{\rho_{G}(x, y): x, y \in D\right\}=$ $\rho_{G}(a, b)$. Let $\varepsilon>0$ be such that $u+B(\varepsilon) \subset G$ for all $u \in D$, where $B(\varepsilon)=\{x \in$ $E:\|x\| \leq \varepsilon\}$. Since $\bar{D}$ is connected, there exist $k \in \mathbb{N}$ and $\gamma_{i}(t)=t u_{i}+(1-t) u_{i+1}$, $i=1, \ldots, k$, such that $u_{i} \in \bar{D}$ for $i=1, \ldots, k+1, a=u_{1}$ and $b=u_{k+1}, u_{i}, u_{i+1} \in U_{i}$ for $i=1, \ldots, k-1$, and $U_{i} \cap U_{j} \neq \varnothing$ iff $|i-j| \leq 1, u_{i} \in U_{i-1} \cap U_{i}$ for $i=2, \ldots, k-1$ and $U_{i}=u_{i}+B(\varepsilon) \subset G$ for $i=1, \ldots, k$; then $M_{D} \leq k \cdot \operatorname{diam}(D)<+\infty$.

We prove that

$$
\left\|f_{n}(x)-f_{n}(y)\right\| \leq \operatorname{diam}(D) \cdot\|\varphi(x)-\varphi(y)\| \quad \text { for all } x, y \in G .
$$

Indeed, suppose that $x, y \in G$. By the Hahn-Banach theorem, there exists $l \in E^{*}$ such that

$$
l\left[f_{n}(x)-f_{n}(y)\right]=\left\|f_{n}(x)-f_{n}(y)\right\| \quad \text { and } \quad|l(u)| \leq\|u\| \quad \text { for all } u \in E .
$$

Let $g_{n}$ be the map defined by the formula

$$
g_{n}(u)=(1 / \operatorname{diam}(D))\left(l \circ f_{n}\right)(u), \quad u \in G .
$$

We may assume without loss of generality that $0 \in D$ to obtain

$$
g_{n} \in H^{\infty}(G), \quad\left\|g_{n}\right\| \leq 1,
$$

and, moreover,

$$
\left|g_{n}(x)-g_{n}(y)\right|=\left|[\varphi(x)-\varphi(y)]\left(g_{n}\right)\right| \leq\|\varphi(x)-\varphi(y)\|\left\|g_{n}\right\| .
$$

From (3.12), using 3.9)-3.11, we get 3.8).

Let $G, G_{1} \subset E$ be domains and let $k: G \rightarrow E$ be a holomorphic map such that $k(G) \subset G_{1}$. Then, by using (3.2) for the domains $G$ and $G_{1}$,

$$
\alpha_{G_{1}}\left[k(x), k^{\prime}(x)(v)\right] \leq \alpha_{G}(x, v), \quad(x, v) \in G \times E .
$$

Indeed, then $k$ induces a map $H^{\infty}(k): H^{\infty}\left(G_{1}\right) \rightarrow H^{\infty}(G)$ by the formula $H^{\infty}(k)(h)=h \circ k, h \in H^{\infty}\left(G_{1}\right)$, and $H^{\infty}(k)$ is linear continuous and $\left\|H^{\infty}(k)\right\| \leq 1$. Therefore the adjoint map $H^{\infty}(k)^{*}: H^{\infty}(G)^{*} \rightarrow H^{\infty}\left(G_{1}\right)^{*}$ is also linear continuous and $\left\|H^{\infty}(k)^{*}\right\| \leq 1$. Let $\varphi: G \rightarrow H^{\infty}(G)^{*}$ and $\psi: G_{1} \rightarrow H^{\infty}\left(G_{1}\right)^{*}$ be the respective evaluation maps. Then the diagram

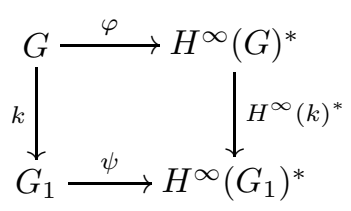


commutes. Consequently,

$$
\begin{aligned}
\alpha_{G_{1}}\left[k(x), k^{\prime}(x)(v)\right] & =\left\|\psi^{\prime}(k(x))\left(k^{\prime}(x)(v)\right)\right\|=\left\|H^{\infty}(k)^{*}\left(\varphi^{\prime}(x)(v)\right)\right\| \\
& \leq\left\|\varphi^{\prime}(x)(v)\right\|=\alpha_{G}(x, v),(x, v) \in G \times E
\end{aligned}
$$

i.e. (3.13) holds.

Suppose that $a \in G$. The set $f_{n}(G)-f_{n}(G)$ is absorbed by $B(\varepsilon)$ (since $f_{n}(G)-$ $f_{n}(G)$ is bounded) and thus there exists $\delta>0$ such that $\delta\left[f_{n}(G)-f_{n}(G)\right] \subset B(\varepsilon)$. Consequently, the map $k_{n}$ defined by the formula

$$
k_{n}(x)=f_{n}(x)+\delta\left[f_{n}(x)-f_{n}(a)\right], \quad x \in G,
$$

is a holomorphic map of $G$ into $G, k_{n}(a)=f_{n}(a)$ and $k_{n}^{\prime}(a)=(1+\delta) f_{n}^{\prime}(a)$. Thus, by (3.13), we have $\alpha_{G}\left[k_{n}(a) ; k_{n}^{\prime}(a) v\right] \leq \alpha_{G}(a ; v)$ for all $v \in E$ and, consequently,

$$
\alpha_{G}\left[f_{n}(a) ; f_{n}^{\prime}(a)(v)\right] \leq \lambda \cdot \alpha_{G}(a ; v) \text { for all } v \in E,
$$

where $\lambda=(1+\delta)^{-1}<1$.

Let $\rho_{G}$ be the integrated form of $\alpha_{G}$ defined by 3.3 . Then $f_{n} \circ \gamma$ is a piecewise $C^{1}$-curve in $G$ from $f_{n}(a)$ to $f_{n}(b)$ and 3.14 implies

$$
\alpha_{G}\left[\left(f_{n} \circ \gamma\right)(t) ;\left(f_{n} \circ \gamma\right)^{\prime}(t)\right] \leq \lambda \cdot \alpha_{G}\left[\gamma(t) ; \gamma^{\prime}(t)\right] \quad \text { for all } t \in\langle 0,1\rangle
$$

Integrating (3.15) on both sides with respect to $t \in\langle 0,1\rangle$, by (3.3), we have

$$
\rho_{G}\left[f_{n}(a), f_{n}(b)\right]=\inf _{\gamma} L\left(f_{n} \circ \gamma\right) \leq \lambda \cdot \inf _{\gamma} L(\gamma)=\lambda \cdot \rho_{G}(a, b)
$$

for all $a, b \in G$.

From (3.2) and (3.3) we get the estimate

$$
\|\varphi(x)-\varphi(y)\| \leq L(\gamma)
$$

for each piecewise $C^{1}$-curve $\gamma$ from $x$ to $y$ in $G$. Thus from (3.8) and (3.3) we get

$$
\left\|f_{n}(x)-f_{n}(y)\right\| \leq \operatorname{diam}(D) \cdot \rho_{G}(x, y) \quad \text { for all } x, y \in G .
$$

Suppose that $a \in G$. For any $n, m \in \mathbb{N}$ and all $b \in G$, by (3.18), (3.16) and (3.4), since the class of holomorphic maps has the composition property, we obtain

$$
\left\|F_{n}(a)-F_{n}(b)\right\| \leq \operatorname{diam}(D) \cdot \lambda^{n-1} \cdot \rho_{G}\left[f_{n}(a), f_{n}(b)\right] \leq \operatorname{diam}(D) \cdot M_{D} \cdot \lambda^{n-1},
$$

$$
\begin{aligned}
\left\|F_{n}(a)-F_{n+m}(b)\right\| & \leq \operatorname{diam}(D) \cdot \lambda^{n-1} \cdot \rho_{G}\left[f_{n}(a),\left(f_{n} \circ f_{n+1} \circ \cdots \circ f_{n+m}\right)(b)\right] \\
& \leq \operatorname{diam}(D) \cdot M_{D} \cdot \lambda^{n-1} .
\end{aligned}
$$

Finally, from (3.20) and (3.4) it follows that, for $b=a$, the sequence $\left\{F_{n}(a)\right\}$ is a Cauchy sequence and since $F_{n}(a)+B(\varepsilon) \subset G$, there exists $w \in G$ such that $\left\{F_{n}(a)\right\}$ converges to $w$. Furthermore, inequalities (3.19) imply $\left\|F_{n}(b)-w\right\| \leq$ $\left\|F_{n}(a)-w\right\|+\operatorname{diam}(D) \cdot M_{D} \cdot \lambda^{n-1}, b \in G$. Consequently,

$$
\eta \stackrel{\forall}{>} 0 n_{0} \stackrel{\exists}{\in} \mathbb{N} n \stackrel{\forall}{\geq} n_{0} b \stackrel{\forall}{\in} G\left\|F_{n}(b)-w\right\|<\eta,
$$

i.e. the sequence $\left\{F_{n}\right\}$ converges to $w$ uniformly on $G$. 


\section{Concluding Remarks}

4.1. If $H^{\infty}(G)$ contains an injective map $g$, then $\rho_{G}$ is metric. Indeed, by the definition of $\varphi$, we have $|g(x)-g(y)|=|[\varphi(x)-\varphi(y)](g)| \leq\|\varphi(x)-\varphi(y)\|\|g\|$. Thus $\varphi$ is injective. A consequence of (3.17) and (3.3) is the inequality $\|\varphi(x)-\varphi(y)\| \leq$ $\rho_{G}(x, y)$ and, by the injectivity of $\varphi$, we get the assertion.

4.2. The conclusion of Theorem 2.1 remains valid if assumptions (a)-(c) are replaced by more general assumption (3.4).

4.3. We derive a short proof of Theorem 2.1 in the special case when $G$ is a nonempty bounded domain. In this case, there exists $R>0$ such that, for any $a \in G$, we have $G \subset B(a, R)=\{x \in E ;\|x-a\|<R\}$. Moreover, we get

$$
\rho_{B(a, R)}(a, x)=\omega[0,\|x-a\| / R]=\sum_{n=0}^{+\infty}[1 /(2 n+1)][\|x-a\| / R]^{2 n+1} \geq\|x-a\| / R,
$$

$x \in B(a, R)$, where $\omega(\cdot, \cdot)$ is a Poincaré metric on $\Delta \times \Delta, \Delta=\{\zeta \in \mathbb{C}:|\zeta|<1\}$. Consequently, for all $a, b \in G$, we have

$$
\rho_{G}(a, b) \geq \rho_{B(a, R)}(a, b) \geq\|b-a\| / R .
$$

From (4.1) and (3.16) we obtain

$$
\begin{aligned}
\left\|F_{n}(a)-F_{n}(b)\right\| & \leq R \cdot \lambda^{n-1} \cdot \rho_{G}\left[f_{n}(a), f_{n}(b)\right] \leq R \cdot M_{D} \cdot \lambda^{n-1}, \\
\left\|F_{n}(a)-F_{n+m}(b)\right\| & \leq R \cdot \lambda^{n-1} \cdot \rho_{G}\left[f_{n}(a),\left(f_{n} \circ f_{n+1} \circ \cdots \circ f_{n+m}\right)(b)\right] \\
& \leq R \cdot M_{D} \cdot \lambda^{n-1} .
\end{aligned}
$$

Further reasoning is analogous. Theorem 2.1]includes the result from [26, Theorem $2.1]$.

4.4. One of the most celebrated theorems of the fixed point theory of holomorphic maps is the theorem of C. J. Earle and R. S. Hamilton 6 which states that each holomorphic map $f: G \rightarrow G$, where $G$ is a bounded domain in $E$ and $f(G)$ lies strictly inside $G$, has a unique fixed point $w \in G$, and $f^{[n]}(a) \rightarrow w$ for any $a \in G$. Their result depends on the fact that $\operatorname{diam}(G)<+\infty$ and one can define a metric on $G$ so that if $f$ maps $G$ into $G$ and is holomorphic, then $f$ is nonexpansive, and if $f$ maps $G$ strictly inside $G$, then $f$ is a strict contraction in this metric.

Theorem 2.1 includes the Earle-Hamilton fixed point theorem [6]. Indeed, maintaining the notation of the previous sections, suppose $G \subset E$ to be a bounded or unbounded domain, $f: G \rightarrow G$ holomorphic and $f(G)$ bounded and lying strictly inside $G$. If $b=f(a)$, then from (3.19) it follows that

$$
\left\|f^{[n]}(a)-f^{[n+1]}(a)\right\| \leq \operatorname{diam}(f(G)) \cdot \lambda^{n} \cdot \rho_{G}(a, f(a)) .
$$

Consequently, $\left\{w_{n}\right\}, w_{n}=f^{[n]}(a), n \in \mathbb{N}$, is a Cauchy sequence and thus converges to some $w \in G$. Since $w_{n}=f\left(w_{n-1}\right)$, therefore, by the continuity of $f$, we obtain $f(w)=w$. If $w^{\prime} \in G$ and $f\left(w^{\prime}\right)=w^{\prime}$, then, by (3.16), we have $\rho_{G}\left(w, w^{\prime}\right)=$ $\rho_{G}\left(f(w), f\left(w^{\prime}\right)\right) \leq \lambda \cdot \rho_{G}\left(w, w^{\prime}\right)$, which implies $\rho_{G}\left(w, w^{\prime}\right)=0$. Furthermore, from (3.18) we then get $\left\|w-w^{\prime}\right\|=\left\|f(w)-f\left(w^{\prime}\right)\right\| \leq \operatorname{diam}(f(G)) \cdot \rho_{G}\left(w, w^{\prime}\right)=0$. Thus $w=w^{\prime}$.

4.5. More recent important results in fixed point theory, concerning the DenjoyWolff Theorem, are established by C.-H. Chu and P. Mellon [3], S. Reich and D. Shoikhet [17] and R. C. Sine [18]. 
4.6. By means of a different set of ideas, I. N. Baker and P. J. Rippon [1, 2], J. Dye, M. A. Khamsi and S. Reich [4], J. M. Dye and S. Reich [5], J. Gill [8]-[11], L. Lorentzen [15] and S. Reich [16] showed the convergence of random iterations of various types of contractions.

\section{REFERENCES}

1. I. N. Baker and P. J. Rippon, On composition of analytic self-mappings of a convex domain, Arch. Math. 55 (1990), 380-386. MR 91k:30062

2. I. N. Baker and P. J. Rippon, Towers of exponents and other composite maps, Complex Variables Theory Appl. 12 (1989), 181-200. MR 91b:30068

3. C.-H. Chu and P. Mellon, Iteration of compact holomorphic maps on a Hilbert ball, Proc. Amer. Math. Soc. 125 (1997), 1771-1777. MR 97g:46060

4. J. Dye, M. A. Khamsi and S. Reich, Random products of contractions in Banach spaces, Trans. Amer. Math. Soc. 325 (1991), 87-99. MR 91h:47003

5. J. M. Dye and S. Reich, Unrestricted iterations of nonexpansive mappings in Banach spaces, Nonlinear Anal. 19 (1992), 983-992. MR 94b:47070

6. C. J. Earle and R. S. Hamilton, A fixed point theorem for holomorphic mappings, Global analysis (Berkeley, Calif., 1968), Proc. Sympos. Pure Math., Vol. 16, Amer. Math. Soc., Providence, RI, 1970, pp. 61-65. MR 42:918]

7. K. Fan, Iteration of analytic functions of operators, Math. Z. 179 (1982), 293-298. MR 83d:47024

8. J. Gill, Complex dynamics of the limit periodic system $F_{n}(z)=F_{n-1}\left(f_{n}(z)\right), f_{n} \rightarrow f$, J. Comput. Appl. Math. 32 (1990), 89-96. MR 92b:58197

9. J. Gill, Compositions of analytic functions of the form $F_{n}(z)=F_{n-1}\left(f_{n}(z)\right), f_{n}(z) \rightarrow f(z)$, J. Comput. Appl. Math. 23 (1988), 179-184. MR 89i:30028

10. J. Gill, Inner composition of analytic mappings on the unit disc, Intern. J. Math. Anal. Math. Sci. 14 (1991), 221-226. MR 92g:30004

11. J. Gill, Limit periodic iteration, Appl. Numerical Math. 4 (1988), 297-308. MR 89f:54061

12. K. Goebel and S. Reich, Uniform convexity, hyperbolic geometry and nonexpansive mappings, Marcel Dekker, New York and Basel, 1984. MR 86d:58012

13. K. Goebel and S. Reich, Iterating holomorphic self-mappings of the Hilbert ball, Proc. Japan. Acad. 58 (1982), 349-352. MR 84b:47065

14. L. A. Harris, Schwarz-Pick systems of pseudometrics for domains in normed linear spaces, Advances in Holomorphy (J. A. Barroso, ed.), North-Holland Math. Stud., vol. 34, Amsterdam, 1979, pp. 345-406. MR 80j:32043

15. L. Lorentzen, Compositions of contractions, J. Comput. Appl. Math. 32 (2) (1990), 169-178. MR 92f:30035

16. S. Reich, The alternating algorithm of von Neumann in the Hilbert ball, Dynamic Systems Appl. 2 (1992), 21-26. MR 94g:47069

17. S. Reich and D. Shoikhet, The Denjoy-Wolff theorem, Ann. Univ. Mariae Curie-Skłodowska 51 (1997), 219-240. MR 2000a:32036

18. R. C. Sine, Behavior of iterates in the Poincaré metric, Houston J. Math. 15 (1989), 273-289. MR 91a:32034

19. A. Stachura, Iterates of holomorphic self-maps of the unit ball in Hilbert space, Proc. Amer. Math. Soc. 93 (1985), 88-90. MR 86b:47117

20. T. J. Suffridge, Common fixed points of commuting holomorphic maps of the hyperball, Michigan Math. J. 21 (1974), 309-314. MR 51:3903

21. E. Vesentini, Iterates of holomorphic mappings, Uspekhi Mat. Nauk 40 (1985), 13-16. MR 87c:58013

22. E. Vesentini, Su un teorema di Wolff e Denjoy, Rend. Sem. Mat. Fis. Milano 53 (1983), 17-25. MR 87m:47137

23. K. Włodarczyk, Iterations of holomorphic maps of infinite dimensional homogeneous domains, Monatsh. Math. 99 (1985), 153-160. MR 86i:46049

24. K. Włodarczyk, Julia's lemma and Wolff's theorem for $J^{*}$-algebras and complex Hilbert spaces, Proc. Amer. Math. Soc. 99 (1987), 472-476. MR 88a:46049 
25. K. Włodarczyk, Studies of iterations of holomorphic maps in $J^{*}$-algebras and complex Hilbert spaces, Quart. J. Math. Oxford 37 (1986), 245-256. MR 87k:46099

26. W. J. Zhang and F. Y. Ren, Random iteration of holomorphic self-maps over bounded domains in $\mathbb{C}^{N}$, Chin. Ann. Math. 16B (1995), 33-42. MR 96d:32034

Faculty of Mathematics, University of Łódź, Banacha 22, 90-238 Łódź, Poland

E-mail address: wlkzxa@imul.uni.lodz.pl

Faculty of Mathematics, University of Łódź, Banacha 22, 90-238 Łódź, Poland

Faculty of Mathematics, University of Łódź, Banacha 22, 90-238 Łódź, Poland 